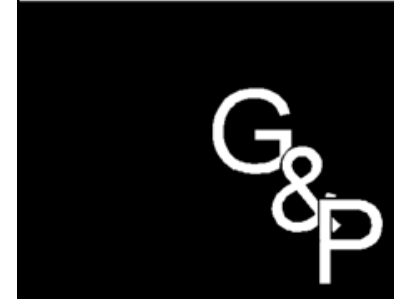

GESTÃO

$\&$

PRODUÇÃO

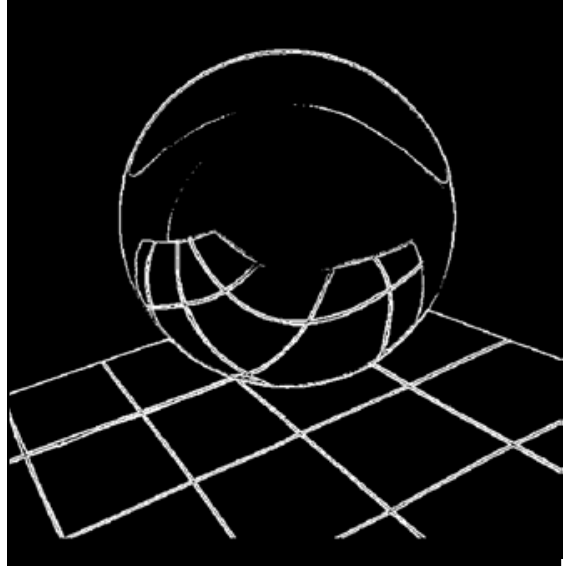

v.6, n.2, p. 87-96, ago. 1999

\section{DETERMINAÇÃO DO NÚMERO ÓTIMO DE CLASSIFICAÇÕES IMPERFEITAS NA AVALIAÇÃO DA CONFORMIDADE DE PRODUTOS}

\author{
Roberto da Costa Quinino \\ Departamento de Estatística - ICEX - UFMG \\ E-mail: roberto@est.ufmg.br \\ Emerson Carlos Colin \\ Pedro Rodrigues Bueno Neto \\ Departamento de Engenharia de Produção \\ Escola Politécnica - USP \\ E-mail: emerson@usp.br
}

\title{
Resumo
}

Neste trabalho estudamos o número ótimo de classificações independentes por unidade produzida supondo que haja dois tipos de erro: o tipo I, no qual classifica-se um produto como não-conforme quando ele na realidade é conforme; e o tipo II, no qual classifica-se um produto como conforme quando na realidade é não-conforme. Desenvolvemos um modelo econômico para minimizar o custo médio total em função dos erros de classificação e do custo de cada classificação. Pela complexidade da função-objetivo associada ao modelo, utilizamos um procedimento exaustivo de busca juntamente com um limitante superior do valor ótimo. Todo o procedimento computacional teve uma implementação relativamente simples e está disponivel como uma planilha eletrônica do Excel e um código do software estatístico Minitab.

Palavras-chave: qualidade, controle por atributos, conformidade, classificações repetidas, erro de classificação, minimização, custo médio total.

\section{Introdução}

$\mathrm{T}$ estes para verificação da qualidade por atributos constituem parte importante da maioria dos processos produtivos. São primordi- ais especialmente quando os processos apresentam características de incontrolabilidade ou o produto final é composto de diversos componentes. Como exemplo de processos em que sempre há verificação de atributos, podemos citar a 
pintura de automóveis, a manufatura de circuitos integrados, a fabricação de discos rígidos para computador e o teste de conjuntos montados como veículos e autopeças.

Os testes de qualidade constituem-se da classificação de cada produto fabricado em conforme ou não-conforme. Estes são normalmente realizados supondo que o sistema de classificação é perfeito, apesar de nem sempre podermos considerar essa hipótese como verdadeira. Além disso, estamos supondo que as classificações não são destrutivas.

BURKE et al. (1995) e GRAMOPADHYE et al. (1996) argumentam que os erros estão longe de serem considerados desprezíveis em muitas tarefas de classificação e podem comprometer seriamente o processo de avaliação da qualidade por atributos. JOHNSON et al. (1991) apresentam numerosos estudos em que verificam que os erros de classificação influenciam seriamente o processo da avaliação da qualidade por atributos.

Neste trabalho tratamos de testes cujas classificações podem apresentar erros. Estamos considerando que dois tipos de erros são possíveis: um é o erro de classificação tipo I, no qual classifica-se um produto como não-conforme sendo na realidade conforme; outro é o erro de classificação tipo II, no qual classifica-se um produto como conforme sendo na realidade nãoconforme. Dessa forma, os testes podem indicar um falso controle da qualidade por atributos. A questão aqui será de como melhorar o desempenho dos testes de qualidade pela diminuição da influência dos erros de classificação.

Uma estratégia que propomos para resolver o problema apresentado é realizar $m(m \geq 0 \quad \mathrm{e}$ inteiro) classificações independentes por produto fabricado e considerar como classificação final do produto a maioria dos resultados obtidos. Tal procedimento, apesar de intuitivo, pode ser inviável economicamente. Essa inviabilidade, conforme veremos adiante, vai depender de uma ponderação entre quanto custa cada classificação, cada envio de produto não-conforme para o mercado e cada produto julgado erroneamente não-conforme. Sendo assim, há necessidade de um modelo que indique qual o número ótimo de classificações independentes repetidas no sentido econômico.

GREENBERG \& STOKES (1995) apresentam um modelo particular, no qual determinam o número ótimo de classificações repetidas considerando apenas a presença do erro tipo I. A hipótese do modelo implica que o "comprador" só recebe produtos realmente conformes e a preocupação da empresa "produtora" é focada em diminuir o número de produtos julgados nãoconformes erradamente. Consideramos que esta premissa não é válida para a maioria dos processos produtivos, uma vez que o erro tipo II é constatado tecnicamente nas usuais reclamações dos "compradores". Sua magnitude é considerada como diferencial importante na seleção de uma empresa "produtora". Neste trabalho apresentamos um modelo para determinação do número ótimo de classificações repetidas independentes dos produtos fabricados, considerando a possibilidade dos erros tipo I e tipo II, e que além disso, pode ser facilmente implementado na prática.

O restante do trabalho é dividido em três seções. A seção 2 descreve o modelo probabilístico adequado. Na seção 3, discutimos o modelo de custo para determinação do número de classificações repetidas independentes e derivamos um limitante superior para determinação do número ótimo de classificações repetidas. Uma aplicação numérica com implementação computacional do modelo proposto é apresentada na seção 4. Finalizamos o trabalho com algumas discussões que podem ser relevantes para novas aplicações e trabalhos futuros.

\section{Modelo Probabilístico}

$\mathrm{C}$ onsidere uma classificação de $n$ produtos em conformes e não-conformes. Seja $p$ a probabilidade de que um produto qualquer seja fabricado conforme, $e_{1}$ a probabilidade de que um produto conforme seja classificado como não-conforme em uma única classificação e $e_{2}$ a probabilidade de que um produto não-conforme 


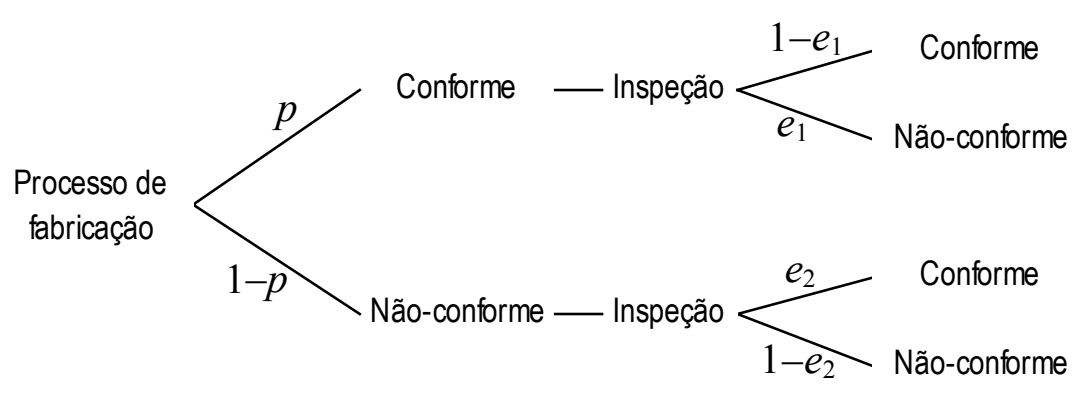

Figura 1 - Árvore de eventos das probabilidades consideradas.

seja classificado como conforme em uma única classificação. A Figura 1 mostra uma árvore de eventos para facilitar a visualização destas probabilidades.

Suponha que cada um dos $n$ produtos seja classificado independentemente $m$ vezes. Considere $C_{i j}(i=1, \ldots, n ; j=1, \ldots, m)$ como uma variável 0 -1, correspondente ao $i j$-ésimo evento, isto é, a $j$-ésima classificação do $i$-ésimo produto; $C_{i}$ também uma variável $0-1$, correspondente à classificação final do $i$-ésimo produto após as $m$ classificações independentes. Por exemplo, $\quad C_{23}=1$ significa que na terceira classificação independente $\mathrm{o}$ produto 2 foi considerado conforme e $C_{3}=1$ significa que o produto 3 foi considerado conforme após as $m$ classificações independentes. Observe a Tabela 1 para uma melhor visualização.

Neste ambiente, estaremos considerando que o $i$-ésimo produto será julgado conforme, $C_{i}=1$, se e somente se $\sum_{j=1}^{m} C_{i j}>0,5 m(i=1, \ldots, n)$. Caso contrário será julgado não-conforme $\left(C_{i}=0\right)$. Observe que em caso de empates nas classificações repetidas, possível quando $m$ é par, o critério de decisão favorece o "comprador" evitando o erro tipo II, considerado neste trabalho, em termos de custos, mais sério que o erro tipo I. A probabilidade de $C_{i}=1$ pode ser expressa por:

$$
\operatorname{Pr}\left[C_{i}=1\right]=\operatorname{Pr}\left[\sum_{j=1}^{m} C_{i j}>0,5 m\right], i=1, \ldots, n .
$$

Suporemos que os resultados tipo Bernoulli $C_{i}$ são independentes e identicamente distribuídos. Definindo $E_{i}(i=1, \ldots, n)$ como uma variável $0-1$, correspondente ao estado real de fabricação do $i$-ésimo produto e utilizando o teorema da probabilidade total podemos expressar $\operatorname{Pr}\left[C_{i}=1\right]$ da seguinte forma:

$$
\begin{aligned}
& \operatorname{Pr}\left[C_{i}=1\right]= \operatorname{Pr}\left[E_{i}=1\right] \operatorname{Pr}\left[C_{i}=1 / E_{i}=1\right]+ \\
&+\operatorname{Pr}\left[E_{i}=0\right] \operatorname{Pr}\left[C_{i}=1 / E_{i}=0\right] \\
&=p\left[1-\sum_{x=0}^{\lfloor 0,5 m\rfloor}\left(\begin{array}{l}
m \\
x
\end{array}\right)\left(1-e_{1}\right)^{x} e_{1}^{m-x}\right]+ \\
&+(1-p)\left[1-\sum_{x=0}^{\lfloor 0,5 m\rfloor}\left(\begin{array}{l}
m \\
x
\end{array}\right) e_{2}^{x}\left(1-e_{2}\right)^{m-x}\right]
\end{aligned}
$$

onde o termo $\operatorname{Pr}[a / b]$ indica a probabilidade condicional de $a$ dado $b$ e $l=\lfloor 0,5 m\rfloor$ indica o maior inteiro inferior ou igual a $0,5 \mathrm{~m}$.

Utilizando a notação $B[a ; b ; c]$ para uma Função Distribuição Binomial, com parâmetros $a$ e $b$, calculada no ponto $l$, temos

$$
\begin{aligned}
\operatorname{Pr}\left[C_{i}=1\right]= & p\left(1-B\left[m ;\left(1-e_{1}\right) ; l\right]\right)+ \\
& +(1-p)\left(1-B\left[m ; e_{2} ; l\right]\right)
\end{aligned}
$$

Para situações reais é razoável assumir que $e_{1}<0,5$ e $e_{2}<0,5$. BURKE et al. (1995) e GRAMOPADHYE et al. (1996) argumentam que $e_{1} \leq 0,25$ e $e_{2} \leq 0,25$ são as situações plausíveis de serem encontradas na prática. Assim sendo, o crescimento de $m$ faz decrescer a 
Tabela 1 - Classificação de $n$ produtos, $m$ vezes cada.

\begin{tabular}{cccccccc}
\hline Produto & \multicolumn{4}{c}{ Classificações $\left(C_{i j}\right)$} & \multirow{2}{*}{ Classificação final $\left(C_{i}\right)$} \\
\cline { 2 - 6 } & 1 & 2 & 3 & $\ldots$ & $m$ & \\
\hline 1 & $C_{11}$ & $C_{12}$ & $C_{13}$ & $\ldots$ & $C_{1 m}$ & $C_{1}$ \\
2 & $C_{21}$ & $C_{22}$ & $C_{23}$ & $\ldots$ & $C_{2 m}$ & $C_{2}$ \\
3 & $C_{31}$ & $C_{32}$ & $C_{33}$ & $\ldots$ & $C_{3 m}$ & $C_{3}$ \\
$\hbar$ & $\hbar$ & $\hbar$ & $\hbar$ & & $\hbar$ & $\hbar$ \\
$n$ & $C_{\mathrm{n} 1}$ & $C_{\mathrm{n} 2}$ & $C_{\mathrm{n} 3}$ & $\ldots$ & $C_{n m}$ & $C_{n}$ \\
\hline
\end{tabular}

probabilidade de julgamentos equivocados sobre a conformidade ou não dos produtos. Neste sentido, com o aumento de $m$, o número de produtos considerados conformes converge para a proporção de produtos fabricados conformes, como podemos observar na proposição 1 .

Proposição 1. Com o aumento de $m$, o número de produtos classificados conformes converge para a proporção de produtos fabricados conformes.

Demonstração. A demonstração pode ser realizada pela aproximação da distribuição Binomial pela distribuição Normal. Assim, temos:

$$
\begin{aligned}
\operatorname{Lim}_{m \rightarrow+\infty} \operatorname{Pr}\left[C_{i}=1\right]= & p-p \Phi\left[\frac{l-m\left(1-e_{1}\right)}{\sqrt{m e_{1}\left(1-e_{1}\right)}}\right]+ \\
& +(1-p)\left(1-\Phi\left[\frac{\left(l-m e_{2}\right)}{\sqrt{m e_{2}\left(1-e_{2}\right)}}\right]\right)
\end{aligned}
$$

$\operatorname{Lim}_{m \rightarrow+\infty} \operatorname{Pr}\left[C_{i}=1\right]=p-p \Phi[-\infty]+(1-p)(1-\Phi[+\infty])=p$

onde $\Phi[\cdot]$ indica função distribuição Normal padrão.

\section{Modelo para Minimização de Custos}

Consideremos $c_{0}$ o custo de classificar um

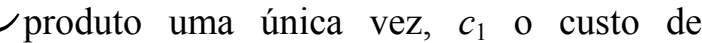

erroneamente julgar um produto conforme como não-conforme e $c_{2} \mathrm{o}$ custo de julgar erroneamente um produto não-conforme como conforme. Se não houver classificações $(m=0)$, o custo médio total $\left(E_{m}\right)$ será definido como

$$
E_{m}\left(p, e_{1}, e_{2}\right)=n(1-p) c_{2}, m=0 .
$$

Em última análise, estamos considerando que toda a produção é conforme e por conseqüência somente o erro tipo II pode estar presente. O número médio de produtos não-conformes no mercado será $n(1-p)$.

Já para $n$ produtos, cada um classificado $m$ vezes ( $m \geq 1$ e inteiro), podemos expressar o custo médio total $\left(E_{m}\right)$ como

$$
\begin{aligned}
E_{m}\left(p, e_{1}, e_{2}\right) & =n m c_{0}+ \\
+ & n \operatorname{Pr}\left[E_{i}=1\right] \operatorname{Pr}\left[C_{i}=0 / E_{i}=1\right] c_{1}+ \\
+ & n \operatorname{Pr}\left[E_{i}=0\right] \operatorname{Pr}\left[C_{i}=1 / E_{i}=0\right] c_{2} \\
E_{m}\left(p, e_{1}, e_{2}\right) & =n m c_{0}+n p B\left[m ;\left(1-e_{1}\right) ; l\right] c_{1}+ \\
& +n(1-p)\left(1-B\left[m ; e_{2} ; l\right]\right) c_{2}, m \geq 1
\end{aligned}
$$

onde $B\left[m ;\left(1-e_{1}\right) ; l\right]=F_{1}$ representa a probabilidade de considerar erroneamente um produto conforme como não-conforme e $1-B\left[m ; e_{2} ; l\right]=F_{2}$ representa a probabilidade de considerar um produto não-conforme como conforme. 
Vamos supor que $m^{\circ}$ seja o $m$ ótimo e que os $m$ válidos pertençam ao conjunto dos inteiros positivos $\left(\mathbf{Z}^{+}=0,1, \ldots\right)$. O problema que estamos considerando se reduz a determinar o $\mathrm{m}^{\circ}$ que satisfaça a equação (2.5) ou semanticamente, o $m^{\circ}$ cujo custo médio total $\left(E_{m}\right)$ seja mínimo, considerando todos os $m$ válidos. Podemos observar que o valor de $m^{\circ}$ independe de $n$.

$$
m^{n}=\arg \min _{m \in \mathbf{Z}^{+}}\left\{E_{m}\left(p, e_{1}, e_{2}\right)\right\}
$$

Em termos operacionais, primeiramente encontramos o $m^{*} \geq 1$ que minimiza (2.4). Em seguida comparamos $\min E_{m \geq 1}\left(p, e_{1}, e_{2}\right) \quad \mathrm{com}$ $E_{m=0}\left(p, e_{1}, e_{2}\right)$. Se $\min E_{m \geq 1}\left(p, e_{1}, e_{2}\right)$ for maior ou igual a $E_{m=0}\left(p, e_{1}, e_{2}\right)$ então $m^{\circ}=0$. Caso contrário $m^{\circ}=m^{*}$. Sendo assim, a grande dificuldade está centrada em se determinar $m^{*}$. Nos desenvolvimentos que se seguem, reduziremos o número de candidatos aptos para $m^{*}$, gerando conseqüentemente condições para determinar $m^{\circ}$.

Para probabilidade de erros de inspeção muito pequena, não é interessante haver inspeções repetidas. Embora intuitivamente correto, fornecemos a prova abaixo mostrando a validade de nosso modelo.

Proposição 2. Se as probabilidades dos erros de classificação são muito pequena, ou seja, $e_{1}, e_{2} \rightarrow 0$, então $m^{*}=1$.

Demonstração. Como $E_{m}\left(p, e_{1}, e_{2}\right)-E_{m}^{*}\left(p, e_{1}, e_{2}\right) \geq 0$ e $0 \leq F_{1}, F_{2} \leq 1$, utilizando (2.4) podemos encontrar que $c_{0}\left(m-m^{*}\right) \geq 0$, e por conseqüência, o único $m^{*}$ que satisfaz a equação é $m^{*}=1$.

Em princípio, desejaríamos encontrar uma forma analítica para $m$ que satisfizesse a equação (2.5) e fosse facilmente implementável computacionalmente. Tentamos utilizar diversos procedimentos convencionais de otimização, entretanto sem sucesso. Em grande parte, a dificuldade acontece devido a equação (2.5) ser definida para valores inteiros de $m$. Além disso, o limite do somatório presente nos cálculos de $F_{1}$ e $F_{2}$ é função de $m$.
Uma solução possível seria escrever de $F_{1}$ e $F_{2}$ como uma função beta incompleta. Tal procedimento provocaria a necessidade de técnicas sofisticadas de cálculo numérico com a conseqüente dificuldade de implementação computacional. Tudo isto nos levou a tentarmos desenvolver procedimentos exaustivos de busca para a localização do $m$ que minimizasse a equação (2.4) e possibilitasse uma implementação computacional simples. Por sua característica intrínseca, os procedimentos exaustivos devem trabalhar juntamente com limitantes, fazendo com que a busca se processe em um intervalo finito de pontos.

Diversos limitantes são possíveis de serem gerados, dependendo das hipóteses assumidas com relação ao modelo e seus parâmetros. A proposição 3 abaixo fornece um limitante bastante aberto, no sentido de que nenhuma hipótese foi considerada na sua elaboração.

Proposição 3. Suponha que $m^{*}$ é o $m$ que minimiza (2.4). Um limitante superior pode ser definido como

$$
m^{*} \leq 1+\left(p c_{1}+(1-p) c_{2}\right) / c_{0} .
$$

Demonstração. Pelo exame de $E_{m}\left(p, e_{1}, e_{2}\right)-$ $E_{m}^{*}\left(p, e_{1}, e_{2}\right) \geq 0$, e após algumas manipulações algébricas com a equação (2.4), podemos obter $m^{*} \leq m+\left(p F_{1} c_{1}+(1-p) F_{2} c_{2}-p F_{1}^{*} c_{1}-(1-p) F_{2}^{*} c_{2}\right) / c_{0}$. Considerando que $0 \leq F_{1}, F_{2} \leq 1$, podemos chegar a $m^{*} \leq 1+\left(p c_{1}+(1-p) c_{2}\right) / c_{0}$.

Das expressões (2.3), (2.4) e (2.6) podemos elaborar uma seqüência de tomada de decisão. Se $p c_{1}+(1-p) c_{2}<c_{0}$ e $c_{0}>(1-p) c_{2}\left(1-\mathrm{e}_{2}\right)-$ $p c_{1} e_{1}$ então $m^{\circ}=0$. Se $p c_{1}+(1-p) c_{2}<c_{0}$ e $c_{0} \leq(1-p) c_{2}\left(1-\mathrm{e}_{2}\right)-p c_{1} e_{1}$ então $m^{\circ}=1$. Agora, se $p c_{1}+(1-p) c_{2} \geq c_{0}$ devemos calcular $E_{m}\left(p, e_{1}, e_{2}\right)$ para todos os valores de $m$, com a restrição de que $m \leq 1+\left(p c_{1}+(1-p) c_{2}\right) / c_{0}$, e considerar $m^{\circ}$ o valor de $m$ que minimiza $E_{m}\left(p, e_{1}, e_{2}\right)$. Para um melhor entendimento mostramos na Figura 2 o fluxograma do processo de tomada de decisão. 


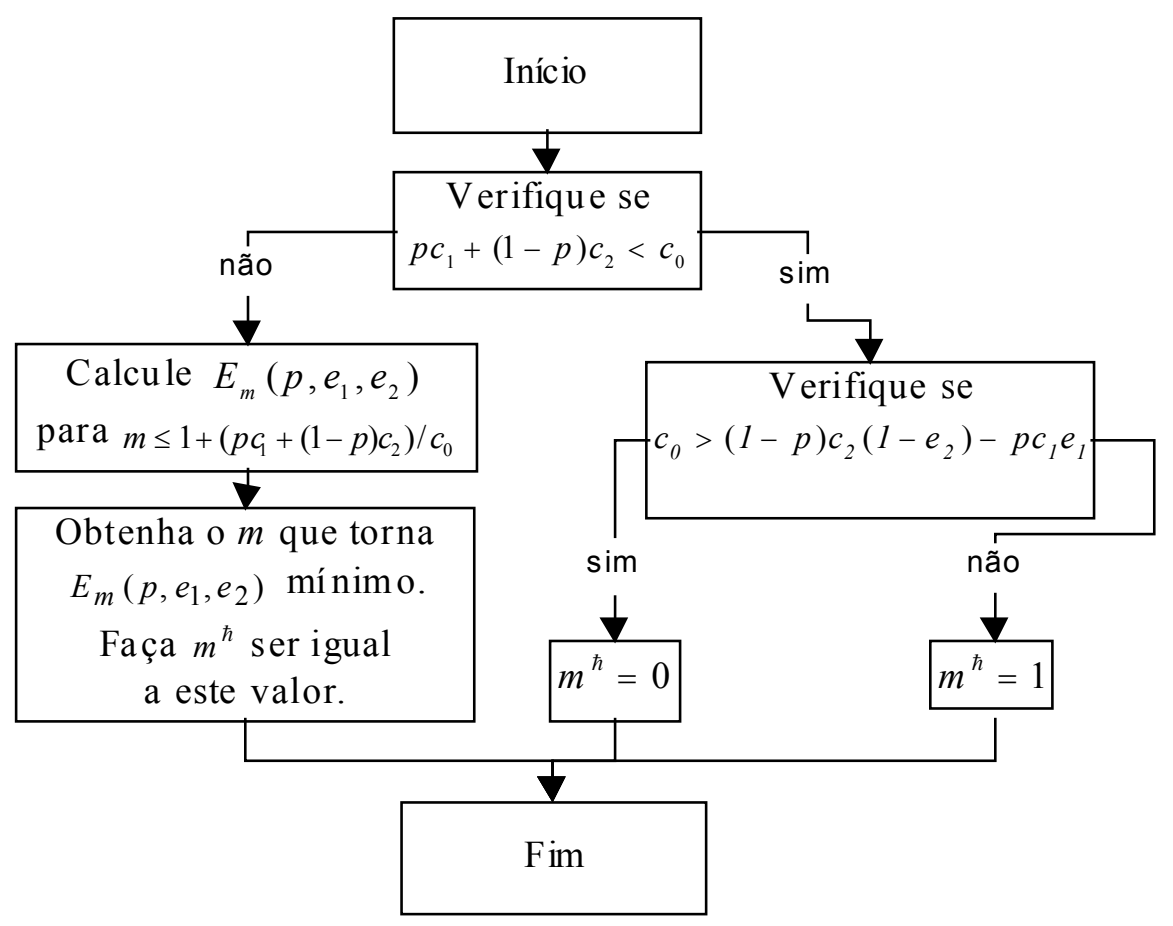

Figura 2 - Fluxograma do processo de decisão.

\section{Aplicação Numérica}

$\mathrm{O}$ exemplo descrito a seguir foi baseado em GREENBERG \& STOKES (1995). Considere uma empresa que fabrica 1000 Circuitos Integrados (CI) por dia. Toda a produção é testada e cada CI é classificado como conforme ou não-conforme. Sabe-se, por meio de experiências práticas, que a classificação de CI pode apresentar erros.

Seja $p=0,95$ a probabilidade de que um CI seja fabricado conforme, $e_{1}=0,1$ a probabilidade de que um CI conforme seja classificado como não-conforme em uma única classificação e $e_{2}=0,1$ a probabilidade de que um circuito integrado não-conforme seja classificado como conforme em uma única classificação.

Os responsáveis pelo setor de planejamento e produção consideram primordial determinar o número ideal de classificações repetidas independentes $(\mathrm{m})$ para os circuitos integrados como forma de minimizar o custo médio total da produção diária. Para tanto, consideram $c_{0}=\$ 1,00, c_{1}=\$ 80,00$ e $c_{2}=\$ 120,00$.
Para o cálculo de $m^{\hbar}$ realizamos implementação computacional no Excel e no software estatístico Minitab. A implementação no Excel é relativamente fácil e não necessita conhecimentos profundos. A Figura 4 do anexo 1 mostra o layout da planilha utilizada. A implementação no Minitab foi realizada pela elaboração de um programa cujo resultado final é o valor de $m^{\circ}$. A Figura 5 do anexo 2 descreve o programa e sua utilização.

A utilização do Excel e/ou do Minitab forneceu o limitante $m^{\circ} \leq 0,83$ gerando o $m$ ótimo $\left(\mathrm{m}^{\circ}\right)$ igual a 3 . O custo médio total foi de $\$ 5296,00$. Observe que o procedimento tradicional de classificar somente uma única vez produz um custo médio total de $\$ 9200,00$. A Figura 3 mostra o comportamento de $E_{m}\left(p, e_{1}, e_{2}\right), \mathrm{m} \geq 0$. A tendência observada na Figura 2 continua para valores de $m$ superiores a 19 .

\section{Conclusão e Considerações Finais}

耳 $\mathrm{m}$ controle por atributos, os erros de classificação podem causar um significativo 


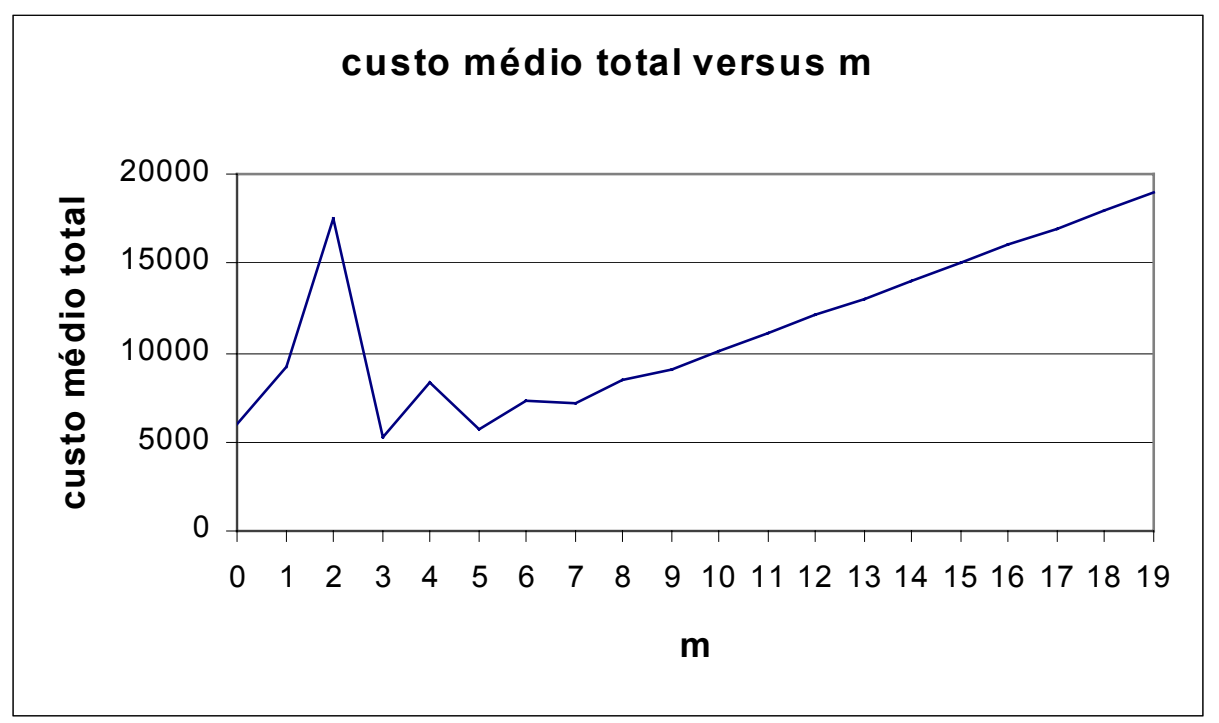

Figura 3 - Custo médio total versus $m$.

impacto nas conclusões sobre a qualidade do processo de produção e conseqüentemente aumentar o custo médio total de todo o processo. Como forma de contornar o problema sugerimos realizar, independentemente, mais de uma classificação por produto fabricado considerando como classificação final do produto a maioria dos resultados obtidos. Tal procedimento mostrou-se capaz de gerar economias sensíveis.

Para tanto, construímos um modelo de minimização dos custos envolvidos. Este mostrounos ser bastante adequado. Além de ser interpretável, possibilitou a determinação de um limitante para o valor que minimiza o custo médio total. Constatamos que todo o processo computacional pode ser resolvido pela implementação computacional simples e com isso tornar-se uma alternativa operacional e padronizada para o processo de produção.
As possibilidades de derivações deste trabalho são muitas. Primeiramente podemos generalizá-lo para o caso de atributos múltiplos. Em segundo lugar, o critério de classificação final dos produtos fabricados pode ser ampliado para $\sum_{j=1}^{m} C_{i j}>a m, \mathrm{i}=1, \ldots, \mathrm{n}$ e $0 \leq \mathrm{a} \leq 1$. Isto gera uma nova função-objetivo para o custo médio total e torna desnecessária as hipóteses $e_{1}<0,5 \mathrm{e}$ $e_{2}<0,5$. Neste sentido, a minimização do custo médio total dependerá de $m$ e $a$. Finalmente podemos considerar $p, e_{1}$ e $e_{2}$ desconhecidos e tratá-los via processo inferencial. Neste sentido, podemos considerar a estatística bayesiana a postura mais adequada. GABA \& WINKLER (1992) apresentam uma inferência bayesiana dos parâmetros $p, e_{1} \mathrm{e} e_{2}$. 


\section{Anexo 1}

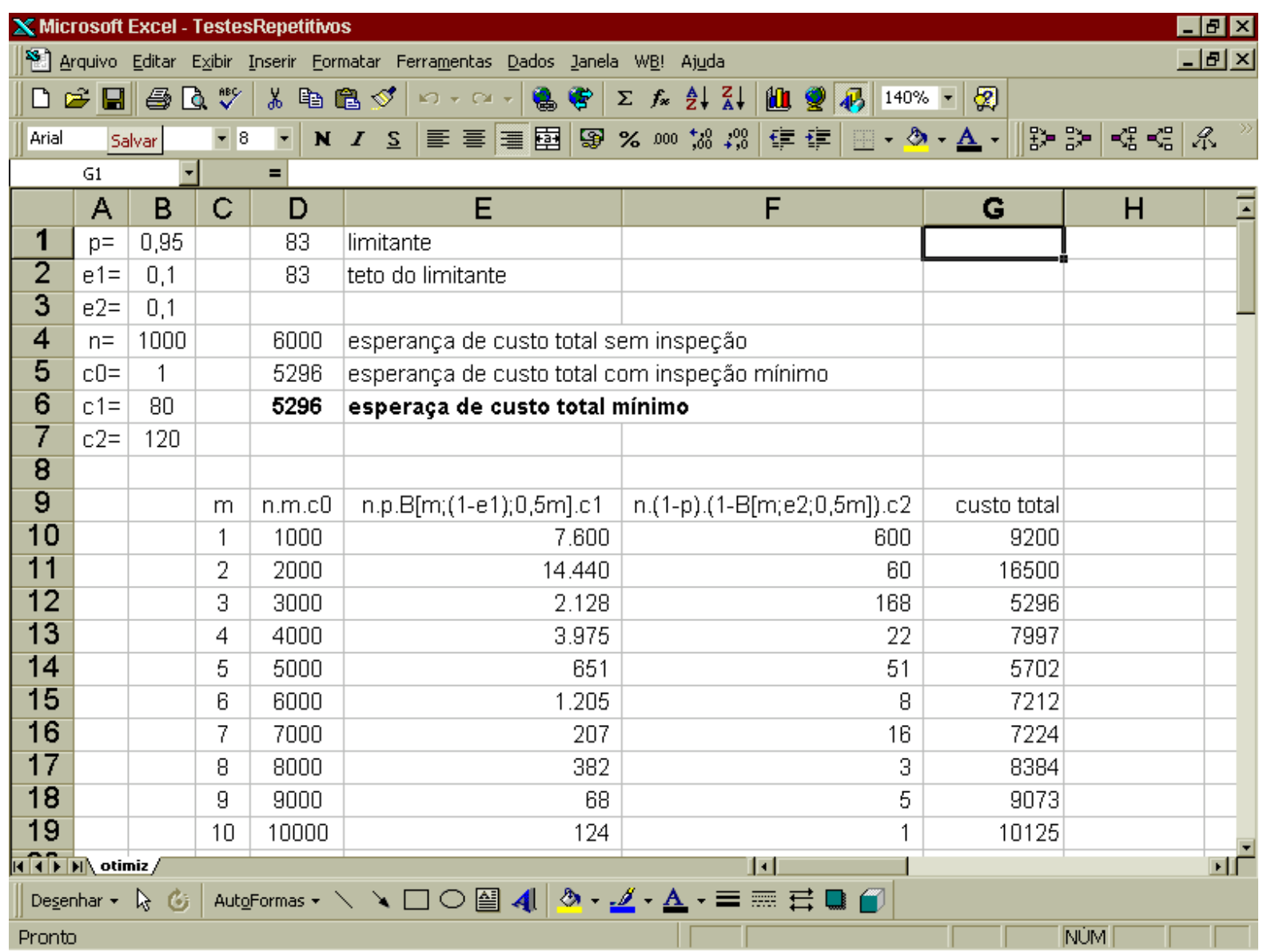

Figura 4 - Planilha Excel para determinação do $m$ ótimo. 


\section{Anexo 2}

Utilize um editor de texto para escrever o arquivo cr.mac. Salve-o, em formato ascii (texto), no diretório mtbwin. Entre no software Minitab. Execute o comando \%cr. Siga as instruções que aparecerem na tela.

\begin{tabular}{|c|c|}
\hline Linhas de Comando & Explicação \\
\hline $\begin{array}{l}\text { gmacro } \\
\text { cr }\end{array}$ & Ativa o modo de programação de macros \\
\hline $\begin{array}{l}\text { note Entre, apertando enter apos cada entrada, } \\
\text { note o Custo c } 0, \mathrm{c} 1, \mathrm{c} 2, \mathrm{p},(1-\mathrm{e} 1),(1-\mathrm{e} 2) \text { e } \mathrm{n} \\
\text { read 'terminal'c } 100 ; \\
\text { nobs } 7 . \\
\text { end } \\
\text { let } \mathrm{k} 1=\mathrm{c} 100(1) \\
\text { let } \mathrm{k} 2=\mathrm{c} 100(2) \\
\text { let } \mathrm{k} 3=\mathrm{c} 100(3) \\
\text { let } \mathrm{k} 4=\mathrm{c} 100(4) \\
\text { let } \mathrm{k} 5=\mathrm{c} 100(5) \\
\text { let } \mathrm{k} 6=\mathrm{c} 100(6) \\
\text { let } \mathrm{k} 6=1-\mathrm{k} 6 \\
\text { let } \mathrm{k} 7=\mathrm{c} 100(7)\end{array}$ & $\begin{array}{l}\text { Entrada dos seguintes valores: } \\
\text { - } c_{0} \rightarrow \text { o custo de classificar um produto uma única } \\
\text { vez. } \\
\text { - } c_{1} \rightarrow \text { custo de erroneamente julgar um produto } \\
\text { conforme como não-conforme } \\
\text { - } c_{2} \rightarrow \text { custo de julgar erroneamente um produto não- } \\
\text { conforme como conforme. } \\
\text { - } p \rightarrow \text { proporção de produtos fabricados conformes. } \\
\text { - }\left(1-e_{1}\right) \rightarrow \text { probabilidade de classificar um produto } \\
\text { conforme como conforme. } \\
\text { - }\left(1-e_{2}\right) \rightarrow \text { probabilidade de classificar um produto } \\
\text { não-conforme como não-conforme. } \\
\text { - } \mathrm{n} \rightarrow \text { número de produtos fabricados. }\end{array}$ \\
\hline $\begin{array}{l}\text { let } \mathrm{k} 8=((\mathrm{k} 4 * \mathrm{k} 2+(1-\mathrm{k} 4) * \mathrm{k} 3) / \mathrm{k} 1)+0.5 \\
\text { round } \mathrm{k} 8 \mathrm{k} 8\end{array}$ & Calcula o limitante para $m^{*}$. \\
\hline $\begin{array}{l}\text { do } \mathrm{k} 9=1: \mathrm{k} 8 \\
\text { let } \mathrm{k} 10=\mathrm{k} 9 / 2 \\
\text { cdf } \mathrm{k} 10 \mathrm{k} 11 ; \\
\text { bino } \mathrm{k} 9 \mathrm{k} 5 \text {. } \\
\text { cdf } 10 \mathrm{k} 12 ; \\
\text { bino } \mathrm{k} 9 \mathrm{k} 6 . \\
\text { Let } \mathrm{k} 12=1-\mathrm{k} 12 \\
\text { let c1 }(\mathrm{k} 9)=\mathrm{k} 11 \\
\text { let c2 }(\mathrm{k} 9)=\mathrm{k} 12 \\
\text { enddo } \\
\text { set c3 } \\
1: \mathrm{k} 8 \\
\text { end } \\
\text { let } \mathrm{c} 4=\mathrm{k} 7 * \mathrm{c} 3 * \mathrm{k} 1+\mathrm{k} 7 * \mathrm{k} 4 * \mathrm{c} 1 * \mathrm{k} 2+\mathrm{k} 7 *(1-\mathrm{k} 4) * \mathrm{c} 2 * \mathrm{k} 3 \\
\text { let } \mathrm{k} 11=\mathrm{k} 7 *(1-\mathrm{k} 4) * \mathrm{k} 3\end{array}$ & Calcula os valores de $E_{m}, \mathrm{~m} \geq 0$. \\
\hline $\begin{array}{l}\text { stack } 0 \text { c3 c3 } \\
\text { stack } \mathrm{k} 11 \mathrm{c} 4 \mathrm{c} 4 \\
\text { sort } \mathrm{c} 3 \mathrm{c} 5 \\
\text { by } \mathrm{c} 4 \text {. } \\
\text { note } \mathrm{O} \text { valor de } \mathrm{m} \text { otimo e: } \\
\text { let } \mathrm{k} 12=\mathrm{c} 5(1) \\
\text { print } \mathrm{k} 12 \\
\text { note } \mathrm{O} \text { custo total esperado ser: } \\
\text { min c4 } \\
\text { endmacro }\end{array}$ & Calcula o valor de $m^{\circ}$ e o custo correspondente. \\
\hline
\end{tabular}

Figura 5 - Programa Minitab para determinação do $m$ ótimo. 


\title{
Referências Bibliográficas
}

BURKE, J.R. et al.: "The effect of inspector errors on the true fraction non-conforming: an industrial experiment", Quality Engineering, 7, pp. 543-50, 1995

GRAMOPADHYE, A.K. et al.: "Compensating for Inspection Errors in Attribute Inspection", Quality Engineering, 8(2), pp. 311-22, 1995-96.
GREENBERG, B.S. \& STOKES, S.L.: "Repetitive testing in the presence of inspection errors", Technometrics, 37(1), pp. 102-11, 1995.

GABA, A. \& WINKLER, R.L.: "Implications of errors in survey data: a bayesian model". Management Science, 38(7), pp. 913-25, 1992.

JOHNSON, N.L.; KOTZ, S. \& WU, X.: "Inspection errors for attributes in quality control", London, Chapman \&Hall, 1991

\section{DETERMINATION OF THE OPTIMAL NUMBER OF IMPERFECT CLASSIFICATIONS IN THE EVALUATION OF THE CONFORMITY OF PRODUCTS}

\begin{abstract}
In this study we investigate the optimal number of independent classifications per unit produced when there are two types of classification errors: type I, where product is classified as conforming when it is not in reality, and type II, where product is classified as non-conforming when in reality it is conforming. We developed an economic model to minimize the total medium cost as a function of the classification errors and the cost of each classification. Due to the complexity of the objective function associated to the model, we used an exhaustive procedure with an upper bound of the optimal value. The procedure may be implemented easily and is available either as an Excel spreadsheet or as a macro of the statistical software Minitab.
\end{abstract}

Key words: quality, control of attributes, conformity, repeated classifications, misclassification, minimization, total medium cost. 